\title{
Gender Inequality on the Uses of the Internet: A Study of Dhaka City of Bangladesh
}

\author{
Jahangir Alam, $\mathrm{MBA}$ \\ Lecturer, School of Business, \\ Ahsanullah University of Science and Technology, Bangladesh
}

Doi:10.19044/esj.2020.v16n23p151 URL:http://dx.doi.org/10.19044/esj.2020.v16n23p151

\begin{abstract}
Nowadays, the internet is considered a basic need, and for this, many researchers and academicians are engrossed by this subject. Very few literary works have been found to show gender inequality on the internet uses. For this reason, this study has been conducted. The main objective of this study is to assess the extent of gender inequalities on the uses of the internet in terms of time spends, money spends, data download, data upload, time spends on Social Networking Sites, time spends per access, and online earnings. For conducting this study, a survey questionnaire has been used for collecting primary data. Six hypotheses are developed and tested at a 5\% level of significance. Results of the study indicate that there is significant inequality that exists between males and females in terms of time spends on the internet; money spends on the internet, the volume of data download and upload, time spends on social networking sites. Besides exists, an insignificant inequality between males and females in terms of time spends on the internet per access. The study recommends that female internet users need to be more thoughtful in case of information access, online earnings, and internet-based government and semi-government services. Based on the findings, the paper recommends that women need awareness on online earnings, online-based government services, and how effectively they can use information. This study suggests ensuring proper uses of the internet for both males and females equally to make Bangladesh competent.
\end{abstract}

Keywords: Gender inequalities; Social networking sites; Online earnings; Internet-based government services; Data download and upload

\section{Introduction}

The internet plays a very vital role in our present generations. It has proved itself a beneficial tool in many ways. In everyday life, the internet is 
playing a very exigent role around the world to gain access to all of the means of the digital age (Pew Research Center Global Attitudes \& Trends, 2017).

The sign of increasing use of the internet has been a good influence in the realms of education, communication, economy, and health sectors (Nazan Dogruer, 2011). Now we can communicate with people across the world through the internet. Despite the uncountable advantages of online internet services in Bangladesh, its scope is mostly underutilized. In April 2013, there was a total of 33,043.124 thousand internet users where the number of male and female users was 23,033.124 and 10,010 thousand, respectively. At the end of June 2020, the total number of internet subscribers has reached 103.476 million. This 103.476 million consists of 94.905 million mobile internet users and 8.571 million are Internet service providers (ISP), and Public switched telephone network(PSTN) users (BTRC, 2020).

A report titled "Mobile, Internet Use: Women far behind men" has published in the daily star and the reporter stated that according to GSMA's 2019 mobile gender gap report $86 \%$ male and 58\% female are using mobile among them only $30 \%$ male and $13 \%$ female are connected to the internet. In Pakistan, $78 \%$ male and 50\% female are using mobile, and among them, 38\% male and $11 \%$ female are connected to the internet, respectively. In Myanmar, $87 \%$ of males and $74 \%$ of females are using mobile, and among them, $57 \%$ male and $35 \%$ female are connected to the internet, respectively (GSMA, Representing the worldwide mobile communications industry, 2019).In Indonesia, $72 \%$ of males and $64 \%$ of females are using mobile, and among them, $43 \%$ male and $36 \%$ female are connected to the internet, respectively. In India, $80 \%$ of males and $59 \%$ of females are using mobile, and among them, $36 \%$ male and $16 \%$ female are connected to the internet, respectively. In China, $96 \%$ of males and $96 \%$ of females are using mobile, and among them, $82 \%$ male and $81 \%$ female are connected to the internet, respectively. (GSMA, The Mobile Gender Gap Report, 2019). Telecom and ICT Minister Mustafa Jabbar said, "Although mobile phone ownership and mobile internet use have increased significantly among women, there is still a persistent gender gap." The honorable minister claimed that the reason behind this scenario is the social structure. He also mentioned that sometimes parents allowed their 12year-old son to use the internet and mobile but not girls even she is 18. But honorable minister upbeat that the scenario will be changed within the next few years. GSMA mentioned that it is necessary to take action by all stakeholders to inform women about the significance of the internet and mobile benefits (Islam, 2018) . From this statistic, apparently, gender disparity is seen in the uses of the internet.

Bangladesh's government dreams that Bangladesh becomes "Digital Bangladesh" by 2021, and by 2021 Bangladesh also becomes a middle-income country. Besides this goal, the internet is also a part of the sustainable 
development goals project. Among 17 SDGs, the internet and technology directly support the $5^{\text {th }}$ and $8^{\text {th }}$ goals, "achieve gender equality and empower all women" and "sustainable economic growth, full and productive employment and decent work for all," respectively. Through ensuring equal internet facility and effective use of the internet, Bangladesh can go forward to achieve these goals (Rahman, 2015).

In Bangladesh, internet users are increasing rapidly. The majority of users are using the internet through mobile phones (BTRC, 2018). Bangladesh's government has taken the initiative to make Bangladesh as Digital Bangladesh and ensure automation of every system, which is one of the prerequisites to build Digital Bangladesh (Silverstone, 2016).To make Digital Bangladesh, it is a cardinal requirement to ensure equal access to the internet for both males and females. Based on this requirement, this study has been done. This paper aims to investigate the difference between males and females on the use of internet in terms of time spends on the internet, money spends, amount of data download \& upload, online earnings, internet-based service experience, and internet uses pattern.

\section{Literature Review}

There are lots of research works that have been found on the internet that uses a pattern. To conduct this research and to find out the research gap, several works of literature have been reviewed. Very few studies focused on gender on the uses of the internet. In human societies, gender is a fundamental principle like class, race, and ethnicity that is widely accepted as a source of inequality. In Bangladesh, the prevailing disparities in areas such as health, education, employment, social and self-steam freedom remain ongoing problems. According to the Human Development Index 2013, Bangladesh was ranked 142 out of 187 countries on the gender index (Hossain, 2005).

According to Weiser (2000), though the internet has been considered as male-dominated, he argued the recent evidence indicates that the gender gap in internet access is rapidly diminishing. But there are some specific differences in the pattern of uses of the internet between males and females. Many gender differences in preferences for particular internet use emerged. Results showed that females use the internet mainly for interpersonal communication and educational assistance. On the other hand, males use it primarily for entertainment and leisure, and some are using to earn money through online activity (Weiser, 2000).

A research was conducted on the gender gap in internet use (martin, 1998), and the researcher found that two-thirds of internet users are male, and the rest are females. The researcher stated that the early exposure and favorable attitude of the male from childhood accounted for the excellent participation in the use of the internet. The researcher also stated that the first 
use of the internet started by male scientists, male hackers, and mathematicians. So, the researcher claimed that this culture is also responsible for lowering participation in internet use. Sometimes, female participation is criticized by the male, and male-dominated online appears. For these reasons, the involvement of females on internet uses is low in comparison with males (Martin, 1998).

A research was conducted by Weiser, (2015) on gender differences in internet use patterns, stated that though internet access is male-dominated, the gender gap in internet use is hastily falling. The researcher used 19 variables to show the gender gap between males and females. The variables are mainly online chat, education, e-mail, shopping, meet new people, research, audio, romance, online games, etc. The result showed that male internet users used the internet for entertainment and leisure purposes. On the other hand, female internet users are using the internet for education purposes, communication purposes, and learning purposes. The study stated that there is a significant difference on internet uses in terms of age of the users.

A study was conducted on "Measuring the Gender Gap on the Internet" by Bimber, University of California, Santa Barbara. The author used the data of three years consecutively and used a logistic regression model on different variables like education, age, income, sex, Latino, race, Black, Asian and others, Housekeeper, married, employed. The gap between male and female are shrinking because of rising tendency of education and economic participation of female. The study showed a significant gender gap exists in internet access. The study claimed that socioeconomic factors are one of the vital factors regarding the gender gap in internet access. Fifty percent digital divide between males and females in internet access is gender-related; besides gender, other factors are also responsible for this gap in internet use (Bimber, 2000). Research showed that internet use accelerates knowledge and communication. The purposes of the internet can help women in various aspects. The study was conducted in China using survey data from 2010 to 2015. The significant findings of the study were that the productive uses of the internet alleviate the biased attitudes against women. The internet enables women to be more sincere and dynamic in their decision making, health, and education perspective (Zhou, Peng, \& Dong, 2019).

A research was conducted on "Towards bridging the grey digital divide," The author used the logistic regression model. The study compares the sample from 2002 to 2014 among different age groups like (40 to 54 years, 55 to 69 years, and 70 to 85 years). The data was used from German ageing groups. The logistic regression model stated that in both periods, the higher age group has lower internet access. The study said that males who are also highly educated were more associated with the internet. Gender and education are significant factors in the case of internet access. The higher income and 
cognitive ability have a positive association in the case of internet access (Oliver Huxhold, 2020).

In Bangladesh, women's access to and use of internet and Information \& Communication Technology tools have been inclusive so far. People claim that women are rather technophobic, and on the other hand, men are much better users of internet and communication technologies. At the same time, many argue that women enthusiastically embrace digital communication (Cimoli, 2010). (Hilbert, 2011) conducted his empirical study on 12 Latin American and 13 African countries from 2005-08 and found the reason of fewer women access and use ICT is a direct result of their unfavorable conditions concerning economic condition, employment, and education. And in case of controlling these types of variables, women turn out to be more efficient users of the internet and digital tools than men. Internet and communication technologies represent a real opportunity to tackle longstanding challenges of gender inequalities in developing countries, including access to health, education, empowerment, and economic activities. Based on the above literature and analysis, the following hypotheses are developed.

$\mathrm{H}_{01}$ : There is no significant inequality between males and females in terms of time spent on the internet.

$\mathrm{H}_{\mathrm{a}}$ : There is significant inequality between males and females in terms of time spent on the internet.

$\mathrm{H}_{02}$ : There is no significant inequality between males and females in terms of money spent on the internet.

$\mathrm{H}_{\mathrm{a}}$ : There is significant inequality between males and females in terms of money spent on the internet.

$\mathrm{H}_{03}$ : There is no significant inequality between males and females in terms of volume of data download.

$\mathrm{H}_{\mathrm{a}}$ : There is significant inequality between males and females in terms of volume of data download.

$\mathrm{H}_{04}$ : There is no significant inequality between males and females in terms of volume of data upload.

$\mathrm{H}_{\mathrm{a}}$ : There is significant inequality between males and females in terms of volume of data upload.

$\mathrm{H}_{05}$ : There is no significant inequality between males and females in terms of time spends on Social Networking Sites.

$\mathrm{H}_{\mathrm{a}}$ : There is significant inequality between males and females in terms of time spends on Social Networking Sites.

$\mathrm{H}_{06}$ : There is no significant inequality between males and females in terms of time spends on the internet per access.

$\mathrm{H}_{\mathrm{a}}$ : There is significant inequality between males and females in terms of time spends on the internet per access. 


\section{The methodology of the study}

This research is quantitative in nature. A questionnaire survey has been used for primary data collection. In the questionnaire, there were 17 questions. There are some basic demographic questions like age, sex, education, profession, etc. Then asked the questions that reflect the basis of gender inequality on the uses of the internet. Different types of variables are used like dichotomous, categorical, and continuous. And the measurement scales of the questionnaire are nominal, ordinal, and ration. This survey has been conducted in Dhaka city, which will reflect the real picture of the study. These primary data are used for further data analysis in this study. Secondary data has been collected to write the literature review and assess the present scenario of gender on the uses of the internet in Bangladesh. The sources of secondary data are different research papers, articles, journals, and other related papers, website information that have been thoroughly studied.

The target population of this paper is those who have access to the internet and resident of Dhaka City Corporation. The sampling frame of this study consists of students, teachers, service holders, and homemakers, and other professions also. In this study, both convenience and purposive sampling techniques have been applied. A total of 250 questionnaires was distributed by using both online and offline methods. One hundred forty complete responses are considered useful for further uses. Among these 140 samples that are considered for further analysis where 70 responses are from males, and 70 are from females. Data are presented and summarized by using Microsoft excel, and for showing gender inequality, six hypotheses are developed based on extensive literature review. Then these hypotheses are tested by using STATA software. STATA 13 version software was used in this research.

\section{Questionnaire Design \& Measurement Scaling}

In the questionnaire, the measurement scales are nominal, ordinal, and ratio, and variables are dichotomous, continuous, and categorical types. The following table shows the items of the questionnaire used for collecting primary through survey along with their measurement scales and the data type.

Table 01: Questionnaire Design \& Measurement Scaling

\begin{tabular}{cllll}
\hline $\begin{array}{c}\text { SL } \\
\text { NO }\end{array}$ & Response Items & $\begin{array}{l}\text { Variable/Data } \\
\text { Type }\end{array}$ & $\begin{array}{l}\text { Measurement } \\
\text { Scale }\end{array}$ & Response Pattern \\
\hline 1 & Gender & Dichotomous & Nominal & Male/Female \\
2 & Age & Continuous & Ratio & Quantitative \\
3 & Profession & Categorical & Ordinal & $\begin{array}{l}\text { Student/Teachers/Service } \\
\text { Holder/Homemaker/Busin } \\
\text { ess/Others }\end{array}$ \\
& & & & $\begin{array}{l}\text { Secondary/Higher } \\
\text { Secondary/Graduation/Po } \\
\text { st-Graduation/Others }\end{array}$ \\
& Educational Level & Categorical & Ordinal & \\
& & & &
\end{tabular}


6

7

8

Amount of Data

download.

10 Amount of Data upload.

11 Amount of time spent in social communication sites.

12 Amount of time spent on the internet per access.

13 Use of internet for online earning purposes.

14 Per month onlin Earnings

15 Online outsourcing is possible nowadays.

16 Experience in internet-based government and semi-government services

17 Intent-based government and semi-government services awareness

\section{Dichotomous Nominal \\ Yes/No}

Continuous

Ratio

Ratio

Ordinal

Categorical

Continuous

Ratio

Continuous Ratio

Continuous Ratio

Quantitative

Quantitative

Information

Access/Communication through SNS/ Information Download/Upload/Merely

Time passing/Plying

Games/Others

Quantitative

Quantitative

Quantitative

Continuous Ratio

Quantitative

Dichotomous Nominal

Yes/No

Quantitative

Continuous Ratio

Yes/No

Nominal

Dichotomous Nominal

Yes/No

Dichotomous Nominal

Yes/No

\section{Data Analysis}

The data analysis and hypotheses testing have been done based on primary data that were collected by using survey questionnaire. In this research, the statistical tools named STATA 13 version software and Microsoft Excel have been used for the analysis of the data. Different tables are used for the demonstration of the analyzed research data. 
Table 02: Demographic characteristics of participants by age

\begin{tabular}{cccccc}
\hline Gender & Age & $\begin{array}{c}\text { Participants } \\
\text { in number }\end{array}$ & $\begin{array}{c}\text { Cumulative } \\
\text { participants } \\
\text { in number }\end{array}$ & Percentage & $\begin{array}{c}\text { Cumulative } \\
\text { percentage }\end{array}$ \\
\hline Male & $15-24$ & 27 & 27 & $39 \%$ & $39 \%$ \\
$(70)$ & $25-34$ & 21 & 48 & $30 \%$ & $69 \%$ \\
& $35-44$ & 16 & 64 & $23 \%$ & $92 \%$ \\
& $45-54$ & 06 & 70 & $08 \%$ & $100 \%$ \\
Total & & 70 & & $100 \%$ & \\
Female & $15-24$ & 20 & 20 & $29 \%$ & $29 \%$ \\
(70) & $25-34$ & 36 & 56 & $51 \%$ & $80 \%$ \\
& $35-44$ & 11 & 67 & $16 \%$ & $96 \%$ \\
Total & $45-54$ & 03 & 70 & $04 \%$ & $100 \%$ \\
\hline
\end{tabular}

Table 2 shows the age category in this study. From the table, it indicates that 140 respondents participated in the study. Among 140 respondents, $39 \%$ male and $29 \%$ female are within the age range of 15-24, $30 \%$ male and $51 \%$ female are from $25-34,23 \%$ male and $16 \%$ female are from $35-44$, and $8 \%$ male and $4 \%$ female are from $45-54$ age range.

Table 03: Demographic characteristics of participants by profession

\begin{tabular}{clcccc}
\hline Gender & \multicolumn{1}{c}{ Profession } & $\begin{array}{c}\text { Participants } \\
\text { in number }\end{array}$ & $\begin{array}{c}\text { Cumulative } \\
\text { participants } \\
\text { in number }\end{array}$ & Percentage & $\begin{array}{c}\text { Cumulative } \\
\text { percentage }\end{array}$ \\
\hline Male & Student & 27 & 27 & $39 \%$ & $39 \%$ \\
$(70)$ & Service Holder & 22 & 49 & $31 \%$ & $70 \%$ \\
& Teacher & 11 & 60 & $16 \%$ & $86 \%$ \\
& Businessmen & 6 & 66 & $09 \%$ & $95 \%$ \\
Total & Others & 4 & 70 & $05 \%$ & $100 \%$ \\
Female & Student & 70 & & $100 \%$ & \\
$(70)$ & Service Holder & 25 & 25 & $36 \%$ & $36 \%$ \\
& Teacher & 13 & 38 & $19 \%$ & $55 \%$ \\
& Businessmen & 12 & 50 & $17 \%$ & $72 \%$ \\
& Homemaker & 0 & 50 & $0 \%$ & $72 \%$ \\
Total & Others & 15 & 65 & $21 \%$ & $93 \%$ \\
\hline
\end{tabular}

Table 3 represents the profession of participants in this study. The table indicates that $39 \%$ of males and $36 \%$ of females are students, $31 \%$ male and $19 \%$ female are service holders, $16 \%$ male, and $17 \%$ female are teachers, $9 \%$ male are doing business. But there is no female business owner, $21 \%$ female are the homemaker, and 5\% male and 7\% female are in others category. These diversified collections of responses may bolster the result of this study. 
Table 04: Demographic characteristics of participants by Educational Qualification

\begin{tabular}{cccccc}
\hline Gender & Education & $\begin{array}{c}\text { Participants } \\
\text { in number }\end{array}$ & $\begin{array}{c}\text { Cumulative } \\
\text { participants } \\
\text { in number }\end{array}$ & Percentage & $\begin{array}{c}\text { Cumulative } \\
\text { percentage }\end{array}$ \\
\hline Male & Secondary & 04 & 04 & $06 \%$ & $06 \%$ \\
$(70)$ & Higher Secondary & 15 & 19 & $21 \%$ & $27 \%$ \\
& Graduation & 29 & 48 & $41 \%$ & $68 \%$ \\
& Post-Graduation & 22 & 70 & $32 \%$ & $100 \%$ \\
Total & Others & 0 & 70 & $0 \%$ & $100 \%$ \\
Female & Secondary & 70 & & $100 \%$ & \\
$(70)$ & Higher Secondary & 11 & 05 & $07 \%$ & $07 \%$ \\
& Graduation & 30 & 46 & $16 \%$ & $23 \%$ \\
& Post-Graduation & 24 & 70 & $43 \%$ & $66 \%$ \\
Total & Others & 0 & 70 & $34 \%$ & $100 \%$ \\
\hline & & 70 & & $0 \%$ & $100 \%$ \\
\hline
\end{tabular}

Table 4 represents the educational qualification of the respondents in this study. Among 70 male participants, 4 or $6 \%$ of participants are secondary school holders, $15(21 \%)$ are higher secondary level, 29(41\%) are graduates while $22(32 \%)$ are postgraduate respondents. Among 70 female participants, 5 or $7 \%$ of participants are secondary school holders, 11(16\%) are higher secondary level, 30(43\%) are graduates while 24(34\%) are postgraduate respondents.

Table 05: Purpose of the internet uses

\begin{tabular}{lcccc}
\hline Purpose of the internet uses & \multicolumn{2}{c}{ Male (70) } & \multicolumn{2}{c}{ Female (70) } \\
\cline { 2 - 5 } & $\begin{array}{c}\text { Positive } \\
\text { Respondents }\end{array}$ & Percentage & $\begin{array}{c}\text { Positive } \\
\text { Respondents }\end{array}$ & Percentage \\
\cline { 2 - 5 } Information Access & 55 & $78 \%$ & 40 & $57 \%$ \\
Social Networking sites users & 61 & $87 \%$ & 64 & $91 \%$ \\
Information Download/Up-load & 38 & $54 \%$ & 29 & $41 \%$ \\
Merely Time passing & 18 & $25 \%$ & 23 & $32 \%$ \\
Plying Games & 19 & $27 \%$ & 09 & $13 \%$ \\
Others & 20 & $28 \%$ & 15 & $21 \%$ \\
\hline
\end{tabular}

The purpose of the internet uses presented in table 5 . The table revealed that $78 \%$ male internet users are using the internet for information access purposes. On the other hand, in the case of female internet users, this number is only $57 \%$. In the case of using social networking sites, $87 \%$ and $91 \%$ there are males and females, respectively, which indicates more females internet social networking sites users than males. 38(54\%) and 29(41\%) male and female respectively used the internet for information download, 18(25\%) males and 23(32\%) females used it for merely time passing while only $19(27 \%)$ males and only 9(13\%) females used it for playing games. In case of information, download and upload 54\% male and $41 \%$ female internet users 
using the internet for information download and upload purposes. $25 \%$ of male and $32 \%$ female internet users using the internet as a merely time passing tool. $19 \%$ of male users are using the internet to play games, but for females, this number is deficient, and the percentage is only $13 \%$. Besides these specific options, there are other purposes to use the internet, with 20(28\%) and $15(21 \%)$ males and females respectively.

Table 06: Internet-based services and online income scenario

\begin{tabular}{|c|c|c|c|c|}
\hline \multirow[t]{2}{*}{ Dimensions } & \multicolumn{2}{|c|}{ Male (70) } & \multicolumn{2}{|c|}{ Female (70) } \\
\hline & $\begin{array}{l}\text { Participants } \\
\text { in number }\end{array}$ & Percentage & $\begin{array}{l}\text { Participants } \\
\text { in number }\end{array}$ & Percentage \\
\hline Having internet access & 70 & $100 \%$ & 70 & $100 \%$ \\
\hline $\begin{array}{l}\text { Knowing that the internet is } \\
\text { using for online outsourcing }\end{array}$ & 68 & $97 \%$ & 52 & $74 \%$ \\
\hline $\begin{array}{l}\text { Using internet for online } \\
\text { earning purpose }\end{array}$ & 15 & $21 \%$ & 4 & $06 \%$ \\
\hline $\begin{array}{l}\text { Received any internet-based } \\
\text { government and semi- } \\
\text { government service receivers }\end{array}$ & 44 & $63 \%$ & 29 & $47 \%$ \\
\hline $\begin{array}{l}\text { Awareness about internet-based } \\
\text { government and semi- } \\
\text { government services }\end{array}$ & 49 & $70 \%$ & 45 & $64 \%$ \\
\hline
\end{tabular}

In table 6 the internet-based services and online income scenario is presented. The study has been conducted who have access to the internet. $68(97 \%)$ of male internet users said that they know that the internet is using or online outsourcing, but the response rate from female internet users is $52(74 \%)$. Though $68(97 \%)$ of male internet users know that internet may use for the online earning purpose only $15(21 \%)$ male internet users are using the internet as an earning tool, on the other hand, this number $4(6 \%)$ in case of female internet users. 44(63\%) male internet users have received any types of internet-based government services; on the other hand, for female internet users, this number is only 29(47\%). 49(70\%) of male internet users are aware of internet-based government and semi-government services, on the other hand, $45(64 \%)$ of female internet users are informed about this issue. It is clear findings that female internet users are lagging in case of using the internet as earning tools, knowledge about online income, internet-based government, and semi-government services.

\section{Hypothesis Testing and Interpretation:}

$\mathrm{H}_{01}$ : There is no significant inequality between males and females in terms of time spent on the internet. 
Table 07: two-sample t-test on time spends on the internet

ttest timespend, by (gender)

Iwo-sample $t$ test with equal variances

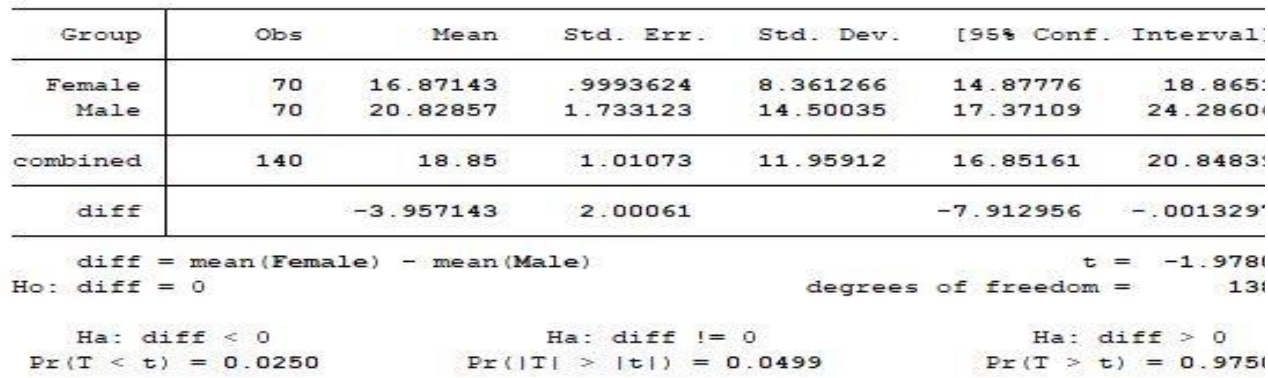

Note: If $\mathrm{p}$-value $<\alpha, \mathrm{H}_{01}$ is rejected at $\alpha$ level of significance. Here $\alpha$ value is 0.05

Table 7 present the two-sample t-test on time spent on the internet by males and females. The table revealed that the p-value is less than the critical value $(p<\alpha(0.0499<0.05)$. This implies that there exists inequality between males and females in terms of time spends on the internet. Therefore, the null hypothesis is rejected. Moreover, the mean value of females and males are 16.87 and 20.82, respectively. This indicates that males spend more time on the internet than females in terms of hours weekly.

$\mathrm{H}_{02}$ : There is no significant inequality between males and females in terms of money spent on the internet.

Table 08: two-sample t-test on money spends on the internet

\begin{tabular}{|c|c|c|c|c|c|c|c|}
\hline Group & & Obs & Mean & Std. Err. & Std. Dev. & [95s conf. & - Interval: \\
\hline $\begin{array}{r}\text { Female } \\
\text { Male }\end{array}$ & & $\begin{array}{l}70 \\
70\end{array}$ & $\begin{array}{l}186.2714 \\
257.6714\end{array}$ & $\begin{array}{l}8.416289 \\
20.28783\end{array}$ & $\begin{array}{l}70.41573 \\
169.7402\end{array}$ & $\begin{array}{l}169 \cdot 4814 \\
217 \cdot 1983\end{array}$ & $\begin{array}{l}203 \cdot 061 ! \\
298 \cdot 144 !\end{array}$ \\
\hline combined & & 140 & 221.9714 & 11.3538 & $134 \cdot 34$ & 199.5229 & $244.419 !$ \\
\hline diff & & & $-71 \cdot 4$ & 21.96429 & & -114.8301 & $-27.9699:$ \\
\hline Ho: diff $\begin{array}{r}\text { diff } \\
\text { Ho: }\end{array}$ & $\begin{array}{l}= \\
=\end{array}$ & mean (Female & b) - mean & le) & degrees & of freedom & $\begin{array}{lr}= & -3.250^{\circ} \\
= & 13 i\end{array}$ \\
\hline $\begin{array}{r}\mathrm{Ha}: \\
\operatorname{Px}(\mathrm{I}<\end{array}$ & & $\begin{array}{l}E f<0 \\
=0.0007\end{array}$ & Pr & $\begin{array}{l}\mathrm{Ha}=\text { diff } \mathrm{I} \\
|>|(\mid)=\end{array}$ & $\begin{array}{l}0 \\
.0014\end{array}$ & $\begin{aligned} H a & =0 \\
\text { Pr }\{(T> & \end{aligned}$ & $\begin{array}{l}\text { diff }>0 \\
\text { t) }=0.999:\end{array}$ \\
\hline
\end{tabular}

Note: If $\mathrm{p}$-value $<\alpha, \mathrm{H}_{02}$ is rejected at $\alpha$ level of significance. Here $\alpha$ value is 0.05

Table 8 present the two-sample t-test on the money spent on the internet by males and females. The table revealed that the $\mathrm{p}$-value is less than the critical value $(\mathrm{p}<\alpha(0.0014<0.05)$. This implies that there exists inequality between males and females in terms of money spends on the internet. Therefore, the null hypothesis is rejected. Moreover, the mean value of females and males are 186.27 and 257.67 , respectively. This indicates that males spend more money on the internet than females in terms of monthly. 
$\mathrm{H}_{03}$ : There is no significant inequality between males and females in terms of volume of data download.

Table 09: two-sample t-test of the volume of data download

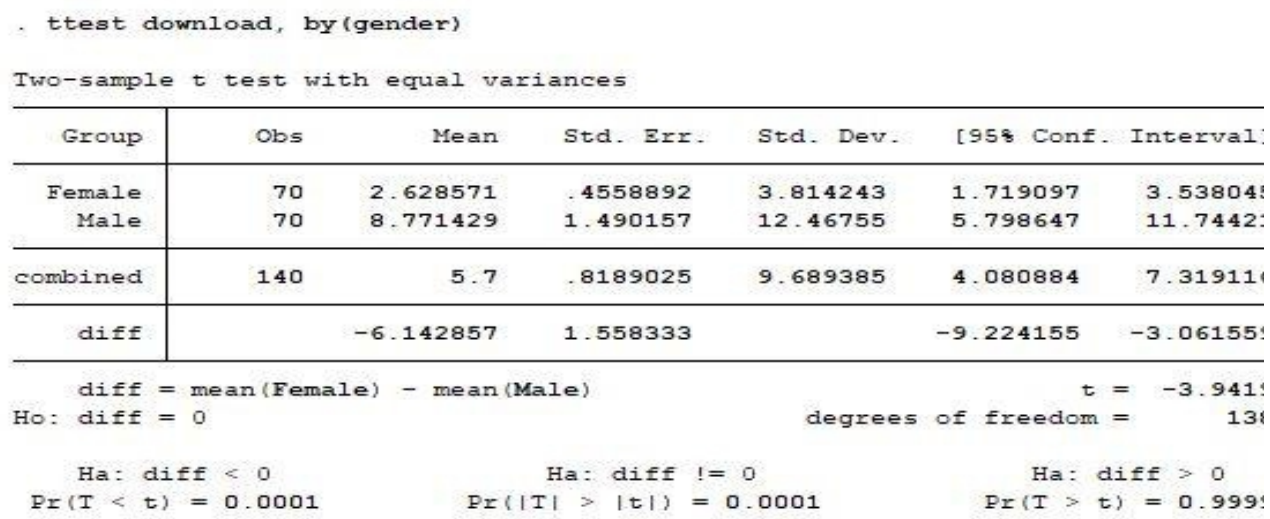

Note: If $\mathrm{p}$-value $<\alpha, \mathrm{H}_{03}$ is rejected at $\alpha$ level of significance. Here $\alpha$ value is 0.05

Table 9 present the two-sample t-test of the volume of data download by males and females. The table revealed that the p-value is less than the critical value $(\mathrm{p}<\alpha(0.0001<0.05)$. This implies that there exists inequality between males and females in terms of the volume of data download. Therefore, the null hypothesis is rejected. Moreover, the mean value of females and males is 2.62 and 8.77, respectively. This indicates that males download more data than females in terms of gigabytes weekly. $\mathrm{H}_{04}$ : There is no significant inequality between males and females in terms of volume of data upload.

Table 10: two-sample t-test of the volume of data upload

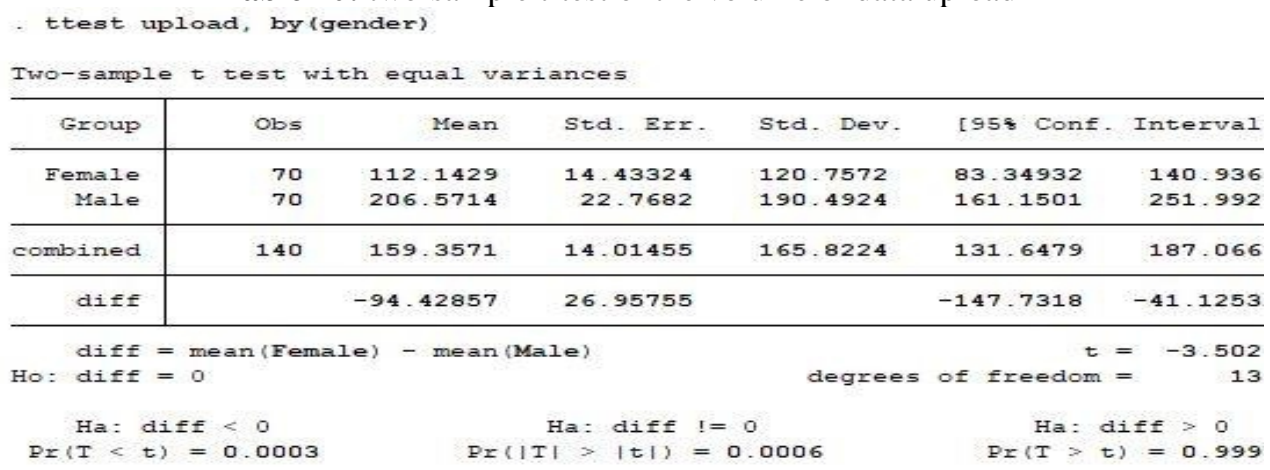

Note: If $\mathrm{p}$-value $<\alpha, \mathrm{H}_{04}$ is rejected at $\alpha$ level of significance. Here $\alpha$ value is 0.05 .

Table 10 present the two-sample t-test of the volume of data upload by males and females. The table revealed that the $p$-value is less than the critical value $(\mathrm{p}<\alpha(0.0006<0.05)$. This implies that there exists inequality between 
males and females in terms of the volume of data upload. Therefore, the null hypothesis is rejected. Moreover, the mean value of females and males is 112.14 and 206.57, respectively. This indicates that males upload more data than females in terms of megabytes weekly.

$\mathrm{H}_{05}$ : There is no significant inequality between males and females in terms of time spends on Social Networking Sites.

Table 11: two-sample t-test of time spends on social networking sites

ttest timeinsns, by (gender)

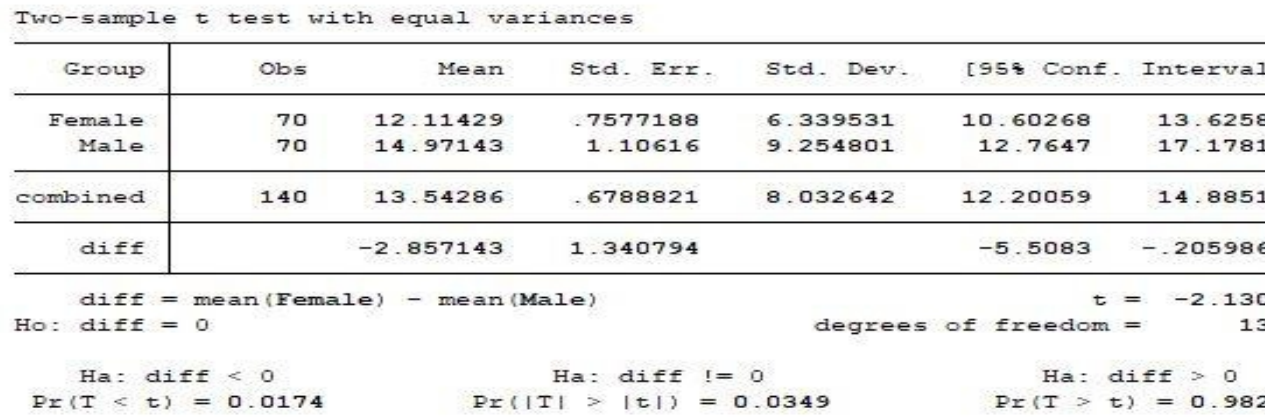

Note: If p-value $<\alpha, \mathrm{H}_{05}$ is rejected at $\alpha$ level of significance. Here $\alpha$ value is 0.05

Table 11 present the two-sample t-test on time spends on social networking sites by males and females. The table revealed that the $\mathrm{p}$-value is less than the critical value $(\mathrm{p}<\alpha(0.0349<0.05)$. This implies that there exists inequality between males and females in terms of time spends on social networking sites. Therefore, the null hypothesis is rejected. Moreover, the mean value of females and males are 12.14 and 14.97, respectively. This indicates that males spend more time on social networking sites than females in terms of hours weekly.

$\mathrm{H}_{06}$ : There is no significant inequality between males and females in terms of time spends on the internet per access.

Table 12: two-sample t-test of time spends on the internet per access

ttest timeperaccess, by (gender)

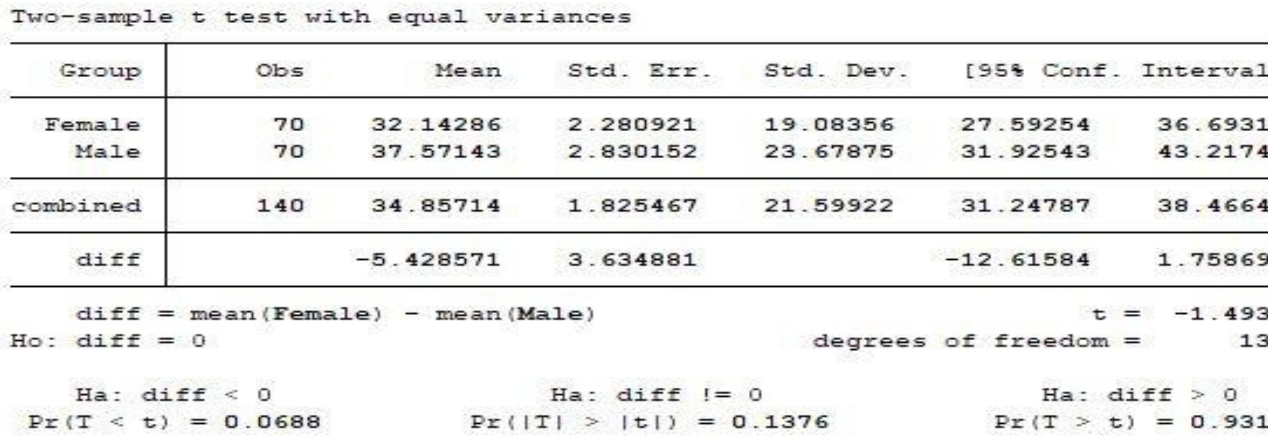

Note: If $\mathrm{p}$-value $<\alpha, \mathrm{H}_{06}$ is rejected at $\alpha$ level of significance. Here $\alpha$ value is 0.05 
Table 12 present the two-sample t-test on time spends on the internet per access by males and females. The table revealed that the $\mathrm{p}$-value is more than the critical value $(\mathrm{p}<\alpha(0.1376<0.05)$. This implies that there exists no significant inequality between males and females in terms of time spends on the internet per access. Therefore, we fail to reject the null hypothesis. Moreover, the mean value of females and males is 32.14 and 37.57, respectively. This indicates that males spend an insignificant time on the internet than females in a single access.

A good number of researchers are working on this issue to boost academic understanding. However, earlier works are showing the gender inequality on the uses of the internet on some general dimensions like age category, access pattern, male-female ratio of uses. But this study specifies the inequalities on specific time spent, money spent, data download and upload, time spent on Social Networking Sites, and time spent per access. Result of this study stated significant differences are existing between male and female on the uses of the internet supported by the works of "gender and the internet" by (Ono, Hiroshi, Zavodny, \& Madeline, 2002), "Measuring the Gender Gap on the Internet" by (Bimber, 2000), "Gendered internet use across generations and life stages" by (Helsper \& Ellen, 2010), "Global internet usage rate 2019, by gender and region" by (J. Clement, 2020), "The digital gender gap is growing fast in developing countries" by ( (ITU, 2019).

\section{Findings and Recommendations}

The findings of this study show that more males than females used the internet for information access (Table 06) whereas, more females than males (Table 06) used the internet for social networking sites. In addition, there are a higher number of male users than females download and upload data (Table 06). For merely time passing purposes, there are more females than males (Table 06) using the internet. More males than females playing games by using the internet (Table 06). 97\% of males and $74 \%$ of females respondents know that the internet is using for online outsourcing (Table 07). More males $(21 \%)$ than females $(06 \%)$ are using the internet for online earning purposes. In the case of internet-based government and semi-government services, more males than females are aware and used to with these facilities (Table 07). Besides, there is significant inequality that exists between males and females in terms of time spends on the internet; money spends on the internet, the volume of data download and upload, time spends on social networking sites (Table 08,09,10,11,12). Also, there exists an insignificant inequality between males and females in terms of time spends on the internet per access (Table 13). In previous works, gender inequality on the uses of the internet showed based on age and general uses purposes. But in this study, the author assessed gender inequality on the uses of the internet very specific way. Like time spends, 
money spends, the volume of data download, volume of data upload, time spends on social networking sites; time spends per access, online earnings, and internet-based government and semi-government services. For that, this study is distinct from previous works.

Nowadays, both formal and informal communication mainly depends on the uses of the internet, like e-mail, Whatsapp, Viber, IMO, messenger, etc. So, both males and females are equally needed to use the internet for effective and efficient communications (Tahri, 2017). Internet is considered a rich communication media; by using the internet, it is possible to contact through audio calls, video calls, group chatting, group video conferencing, and many more (TREVINO, LENGEL, \& DAFT, 1987). If it is possible to ensure internet access and proper uses of the internet for both males and females, then it will facilitate online communication, social communication, group intimacy, and virtual face to face communication. Besides, it also helps orally disable people for non-verbal communication (Short, Williams, \& Christie, 1976).

Both males and females are required to move forward at the same pace to fulfil the dream of Digital Bangladesh. And for this, both must use the internet equally and adequately. More female internet users must be cautious so that they can use the internet for information access instead of social networking sites only. Besides these, female internet users need to increase the use for data download and upload purpose, online outsourcing purpose, internet-based government, and semi-government services purpose. The equal and proper utilization of the internet is required for both males and females; otherwise, the mission and objectives of digital Bangladesh will be a challenge to reach.

\section{Limitations and Future Research}

There are some limitations identified in this study. Firstly, though the participants of both males and females are equal, the sample size is small, and therefore the result cannot be generalized even when the purposive sampling method was used. Second, the study has been conducted in Dhaka city that is unable to represent the whole scenario of the country. With full emphasis, this cannot be a good representative of the population of Bangladesh; therefore, as proper discrimination-free internet uses is a vital factor in coping up with the present world. Future research should be done with enough sample size along with the participation from the whole country so that the real picture of the internet uses can be determined.

\section{Conclusion}

The study investigated the difference between males and females on the uses of the internet in terms of time spends on the internet, money spends on the internet, amount of data download and upload, online earnings, internet- 
based service, and internet use patterns. The findings of this study revealed that though both males and females are using the internet, the significant differences are existing. Females, along with the male need to be cautious in case of productive internet uses. More females should engage themselves in online outsourcing activities. As today's world is impossible to think without proper internet uses, both males and females are required to use the internet appropriately to make Bangladesh as digital Bangladesh in full swing.

\section{References:}

1. Bangladesh Telicommunication Regulatory Communication. (2017, August Monday). Retrieved from http://www.btrc.gov.bd/content/internet-subscribers-bangladeshaugust-2017

2. Bimber, B. (2000). Measuring the Gender Gap on the Internet. SOCIAL SCIENCE QUARTERLY.

3. BTRC. (2017). Retrieved from Bangladesh Telecommunication Regulatory Commission: http://www.btrc.gov.bd/

4. BTRC. (2020, July wednesday). Bangladesh Telecommunication Regulatory Commission (BTRC) . Retrieved from http://btrc.gov.bd/

5. Cimoli, M. H. (2010). The Impact of Information and Communication Technologies in Latin America. United Nations Economic Commission for Latin America and the Caribbean.

6. GSMA. (2019, april 25). Retrieved from Representing the worldwide mobile communications industry: https://www.gsma.com/

7. GSMA. (2019). The Mobile Gender Gap Report. London: gsma association.

8. Hilbert, M. (2011). Digital gender divide or technologically empowered women in developing countries? Women's Studies International, 479-489.

9. Hossain, C. A. (2005). Closing the gender gap in Bangladesh: inequality in education, employment and earnings. International Journal of Social Economics, 439-453.

10. Islam, M. Z. (2018, march 28). Retrieved from The Daily Star: https://www.thedailystar.net/business/telecom/news/mobile-internetuse-women-far-behind-men-1711996

11. ITU. (2016, October). Retrieved from Committed to connecting the world: http://www.internetlivestats.com/internet-users/bangladesh/

12. ITU. (2019). The digital gender gap is growing fast in developing countries. Retrieved from https://itu.foleon.com/itu/measuring-digitaldevelopment/gender-gap/ 
13. Martin, J. M. (1998). The Gender Gap in Internet Use: Why Men Use the Internet More Than Women-A Literature Review. CyberPsychology \& behavior.

14. Nazan Dogruer, R. E. (2011). The use of the internet for educational purposes. ELSEVIER, 606-611.

15. Oliver Huxhold, E. N. (2020). Towards bridging the grey digital divide: changes in internet access and its predictors from 2002 to 2014 in Germany. European Journal of Ageing .

16. Ono, Hiroshi, Zavodny, \& Madeline. (2002). Gender and the Internet . Federal Reserve bank of Atlanta. Retrieved from : Gender and the Internet, Working Paper, No. 2002-10, Federal Reserve Bank of Atlanta, Atlanta, GA.

17. Pew Research Center Global Attitudes \& Trends. (2017, October Tuesday). Retrieved from Pew Research Center: http://www.pewglobal.org

18. Rabayah, k. (2010). Economic and social Empowerment of Women through Information and Communication Technology. Journal of Community Informatics.

19. Rahman, L. (2015, March). Digital Bangladesh: Dreams and reality. Retrieved from http://bangladeshxclusive.blogspot.com/p/digitalbangladesh-vision-2021.html

20. Saroj, K. (2015). Impact of information technology on financial empowerment of women. Journal of science and Management.

21. Short, J., Williams, E., \& Christie, B. (1976). The social psychology of telecommunications. London; New York: Wiley.

22. Silverstone, R. a. (2016). Design and the domestication of information and communication technologies: technical change and everyday life. England: Oxford University Press.

23. Tahri, W. (2017). Choice, use and appropriation of email:an ever evolving technology. Information Systems Management \& Innovation, 4-22.

24. TREVINO, L. K., LENGEL, R. H., \& DAFT, R. L. (1987). Media Symbolism, Media Richness, and Media Choice in Organizations: A Symbolic Interactionist Perspective. Sage Journals, 553-574.

25. Weiser. (2000). Gender Differences in Internet Use Patterns and Internet Application Preferences: A Two-Sample Comparison. CVBER PSYCHOLOGY \& BEHAVIOR.

26. Weiser, E. B. (2015). Gender Differences in Internet Use Patterns and Internet Application Preferences: A Two-Sample Comparison. CVBER PSYCHOLOGY \& BEHAVIOR.

27. Zhou, D., Peng, L., \& Dong, Y. (2019). The impact of Internet usage on gender role attitude. Applied Economics Letters. 
Research Questionnaire

\section{Appendix:}

Dear Participant,

I am Jahangir Alam, Lecturer of School of Business of Ahsanullah University of Science and Technology. I am doing research titled "Gender on the Uses of the Internet: A Study of Dhaka City of Bangladesh." I need your valuable feedback to complete my research work. The survey will only take about 5-7 minutes of your time. Your answers will remain anonymous, do not put your name on the questionnaire. All responses will be kept confidential. Your help in this resarch is strictly voluntary. You do not have to answer any questions if you don't want to. Return of an answered survey will indicate your consent to participate in this study. It is my humble request to you to cooperate so that I can accomplish my research work. If you have questions or concerns, please contact me at jahangir.sob@aust.edu.

Thank you for your time and consideration.

Sincerely

$$
\text { Lalam }
$$

Jahangir Alam

Lecturer

School of Business, Ahsanullah University of Science and Technology (AUST)

\section{QUESTIONNAIRE (প্রম্নপত্র)}

Questionnaire Number:

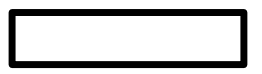

1. Gender: Male (পুরুষ) $\square \quad$ Female (মহিলা)

2. Age (বয়স):

\section{Profession:}

$\square$ Student (ছাত্রছাত্রী)

$\square$ Teachers (শিক্ষক/শিক্ষিকা)

$\square$ Service Holders (পেশাজীবি)

$\square$ Housewives (গৃহিণী)

$\square$ Business (ব্যবসায়) 
$\square$ Others (অन्্ान्্):

4. Educational Qualification:

$\square$ Secondary (মাধ্যমিক)

$\square$ Higher Secondary (ঊচ্চ মাধ্যমিক)

$\square$ Graduation (স্নাতক)

$\square$ Post-Graduation (স্নাতকোত্তর)

$\square$ Others (অন্যান্য):

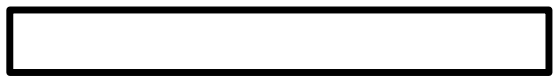

5. Do you Have access to the internet? [If yes, please proceed to the next questions] (আপনার কি ইন্টারনেট সংযোগ আছে) [যদি থাকে, অনুগ্রহ পূর্বক পরবর্তী প্রশ্নে অগ্রসর হোন]
$\square$ Yes (2्্যा)
$\square$ No (না)

6. How much time do you spend on the internet weekly?

(প্রতি সপ্তাহে আপনি কত ঘণ্টা সময় ইন্টারনেট ব্যয় করেন)

\section{Hours/Weekly}

7. How much money do you spend on the internet monthly? (ইন্টারনেট বাবদ মাসিক আপন কত খরচ করেন?)

8. What are the purposes of your internet use? [You may select more than one.] (আপনি কি কি উদ্দেশ্যে ইন্টারনেট ব্যবহার করেন? )[একাধিক উত্তর গ্রহনযোগ্য]

$\square$ Information access (তথ্য অনুপ্রবেশ)

$\square$ Communication Through Social Networkings sites (সামাজিক যোগাযোগ মাধ্যম)

$\square$ Information Download/upload (তথ্য ডাউনলোড/ আপলোড)

$\square$ Merely Time Passing (শুধু সময় কাটানোর জন্য)

$\square$ Plying Games (গেম খেলা)

$\square$ Others (অন্যান্য): 
9. How much data you have download weekly? [Give answer in megabyte or gigabyte] (স্পেতাহে আপনি কি পরিমান ডাটা ডাউনলোড করেন) [উত্তর মেগাবাইট অথবা গিগাবাইটে প্রদান করুন]

$\square$ Weekly/gigabyte (GB) (সাপ্তাহিক/গিগাবাইট)

$\square$ Weekly/megabyte (MB) (মাসিক/ মেগাবাইট)

No Download (কোন ডাউনলোড হয় না)

10. How much data you have upload weekly? [Give answer in megabyte or gigabyte] ((সপ্তাহে আপনি কি পরিমান ডাটা আপলোড করেন) [উত্তর মেগাবাইট অথবা গিগাবাইটে প্রদান করুন]

$\square$ Weekly/gigabyte (GB) (সাপ্তাহিক/গিগাবাইট)

$\square$ Weekly/megabyte (MB) (মাসিক/ মেগাবাইট)

No Download (কোন ডাউনলোড হয় না)

11. How much time do you spend on social communication sites like Facebook, Twitter, WhatsApp, Viber, and YouTube weekly? (আপনি সামাজিক যোগাযোগ মাধ্যমে- ফেসবুক, টুইটার, হোয়াটসঅ্যাপ, ভাইবার, ইউটিউব-এ কি পরিমান সময় ব্যয় করেন)

Hours/Weekly (সাপ্তাহিক/ঘন্টা) :

12. How much time do you spend on the internet per access? [Give answer in minutes or hours] (প্রতিবার একসেসে আপনি ইন্টারনেটে কি পরমান সময় ব্যয় করেন)

$\square$ Minutes (মিনিট)

$\square$ Hours (ঘন্টা)

13. Do you use the internet for online earning purposes? (অনলাইনে আয়ের জন্য আপনি ইন্টারনেট ব্যবহার করেন কি?)

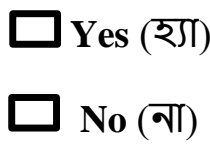

14. If the answer to question number 13 is yes, then what is the average amount you earn per month? (যদি ১৩ নং প্রক্নের উত্তর হ্যা হয়, তবে আপনি মাসিক কত আয় করেন?)

BDT/Monthly (টাকা/মাসিক) 
15. Do you know that using online internet outsourcing is possible nowadays? (ইদানিং ইন্টারনেটের মাধ্যমে আউটসোরসিং করা যায় এ সম্পর্কে আপনি জানেন কি?)
$\square$ Yes (2्্যा)
$\square$ No (না)

16. Do you receive any internet-based government and semi-government services?

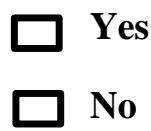

17. Are you aware about intent-based government and semi government services? (আপনি কি ইন্টারনেট ভিত্তিক সরকারী বা আধা সরকারী কোন সেবা সম্পর্কে আবগত?)
$\square$ Yes (2्্যা)
$\square$ No (না)

Thank you for your valuable feedback. (আপনার মূল্যবান মতামতের জন্য ধন্যবাদ 